\title{
Quantum calculation of vortices in the inner crust of neutron stars
}

\author{
P. Avogadro, ${ }^{1,2}$ F. Barranco, ${ }^{3}$ R. A. Broglia, ${ }^{1,2,4}$ and E. Vigezzi ${ }^{2}$ \\ ${ }^{1}$ Dipartimento di Fisica, Università degli Studi di Milano, via Celoria 16, I-20133 Milano, Italy \\ ${ }^{2}$ INFN, Sezione di Milano, via Celoria 16, 20133 I-Milano, Italy \\ ${ }^{3}$ Departamento de Fisica Aplicada III, Escuela Superior de Ingenieros, \\ Camino de los Descubrimientos s/n, E-41092 Sevilla, Spain \\ ${ }^{4}$ The Niels Bohr Institute, University of Copenhagen, Blegdamsvej 17, DK-2100 Copenhagen $\emptyset$, Denmark \\ (Received 26 January 2006; revised manuscript received 26 June 2006; published 12 January 2007)
}

\begin{abstract}
The self-consistent mean-field quantum mechanical solution of a vortex and a nucleus immersed in a sea of free neutrons, a scenario representative of the inner crust of neutron stars, is presented for the first time. Because of quantal size effects the phase space for vortices inside the nucleus is essentially zero, so that the vortex core opens up and surrounds the nucleus. As a consequence, pinned configurations (in which a vortex becomes anchored to the nucleus) are favored at low and high densities in the inner crust. This result is qualitatively different from that obtained in all previous models, which predict pinning at intermediate densities.
\end{abstract}

In the inner crust of a neutron star atomic nuclei are immersed in a sea of free superfluid neutrons permeated by an array of vortices. It has been proposed that the glitches, sudden irregularities in the rotation rate observed in many neutron stars, can be viewed as "vorticity jumps," equivalent to "flux jumps" in a superconducting magnet [1]. To make quantitative comparisons with existing data, one needs to develop detailed models of vortex line configurations and of vortex dynamics (see, e.g., Ref. [2]). In turn, the latter crucially depends on whether the vortex anchors itself to the nuclei or not, a phenomenon controlled by the so-called pinning energy. For example, one needs to know the pinning energy as a function of density to address the question of the global stability of a pinned lattice, and to interpret precession observations [3].

To make a reliable calculation of the pinning energy two questions are to be answered: 1. How important are quantal, finite-size (proximity) effects?, 2. Which role is played by polarization effects on the pairing gap of the system? Although a quantitative estimate of the pinning energy requires that one gives an answer to both questions, qualitatively the two issues are quite independent of each other.

In the following, we present the results of a calculation of the vortex-nucleus interaction in the inner crust that represents a clear improvement on previous work [4-6], because (a) it is based on quantal mean-field theory (Ref. [4] was based on Ginzburg-Landau equations, Ref. [5] used a fixed WoodsSaxon potential, whereas Ref. [6] was based on a semiclassical approximation) and (b) it only assumes axial symmetry for the neutron density (Ref. [5] assumed cylindrical symmetry). Our findings turn out to be qualitatively different from all previous ones. In particular, by comparing them with those obtained within the framework of the semiclassical approximation [6], we are able to answer for the first time the aforementioned question 1.

Arguably, the most accurate (and recent) answer to question 2 indicates that polarization effects play a minor role, being much smaller than previously estimated [7]. In keeping with this result, we have used a pairing interaction that reproduces the pairing gap calculated with a bare force in uniform neutron matter.

Our calculations were performed by solving the mean-field Hartree-Fock-Bogoliubov (HFB) equations (often called the De Gennes equations), within a cylindrical box of radius $30 \mathrm{fm}$ and height $40 \mathrm{fm}$, imposing that the wave functions vanish at the border of the cell. We have solved the vortex-nucleus system in an axially symmetric basis, with the vortex directed along the $z$ axis. The De Gennes equations have the form $[5,8]$

$$
\begin{aligned}
{\left[K+V(\rho, z)-E_{F}\right] U_{\alpha}+\Delta V_{\alpha} } & =E_{\alpha} U_{\alpha}, \\
-\left[K+V(\rho, z)-E_{F}\right] V_{\alpha}+\Delta^{*} U_{\alpha} & =E_{\alpha} V_{\alpha}
\end{aligned}
$$

and have to be solved simultaneously with the number equation. The kinetic energy operator is denoted by $K ; V$ is the self-consistent Hartree-Fock mean field, which was determined using the SII force [9]. The $(S=0)$ pairing field $\Delta$ and the quasiparticle amplitudes $U_{\alpha}$ and $V_{\alpha}$ depend on the coordinates $z, \rho$ (the distance to the $z$ axis in the $x-y$ plane) and $\phi$ (the azimuthal angle). Equations (1) allow for different solutions, which can be labeled by the vortex number $v(=0,1,2 \ldots)$. For a given value of $v$, the pairing gap depends on $\phi$ according to $\Delta(\rho, z, \phi)=\Delta(\rho, z) e^{i \nu \phi}$, and each Cooper pair carries $v$ units of angular momentum along the $z$ axis. For $v=0$ one recovers the usual equations for the superfluid ground state. The quasiparticle amplitudes are expanded on a basis of (free) single-particle wave functions:

$$
\begin{aligned}
& U_{\alpha}(\rho, z, \phi)=e^{i l_{\alpha} \phi} \sum_{n, m} U_{\alpha}^{n, m} \psi_{n, l_{\alpha}}(\rho) \chi_{m}(z), \\
& V_{\alpha}(\rho, z, \phi)=e^{i\left[l_{\alpha}-\nu\right] \phi} \sum_{n, m} V_{\alpha}^{n, m} \psi_{n, l_{\alpha}-\nu}(\rho) \chi_{m}(z),
\end{aligned}
$$

where the functions $\chi_{m}(z)$ are (longitudinal) plane waves and $\psi_{n, l_{\alpha}}(\rho)$ (radial) Bessel functions, with $l_{\alpha}$ being the single-particle angular momentum along the cylinder axis, chosen as quantization axis. The quantities $U_{\alpha}^{n, m}$ and $V_{\alpha}^{n, m}$ are determined by substituting Eqs. (2) into Eqs. (1). This 

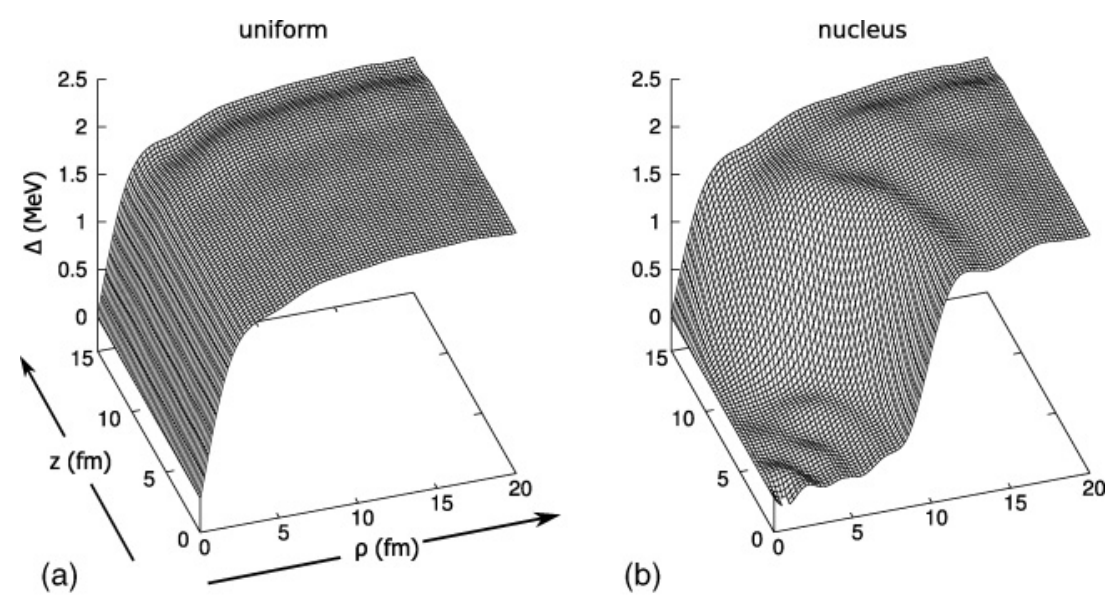

(b)

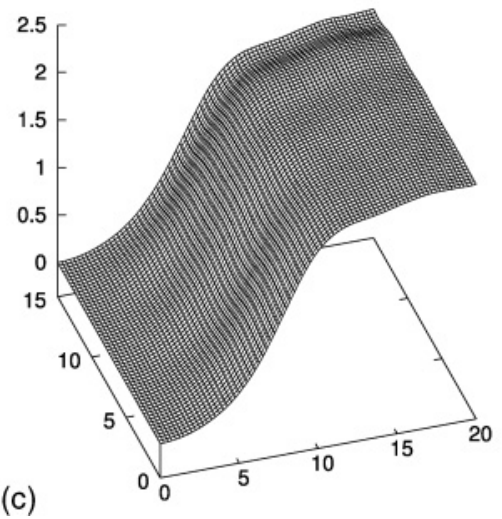

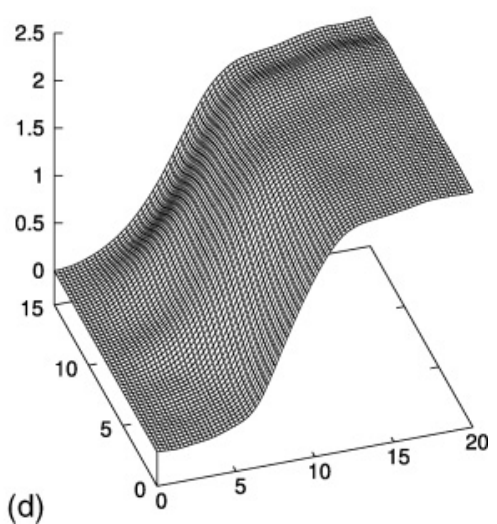

FIG. 1. Pairing gap associated with a $v=1$ and a $v=2$ vortex, calculated in the cylindrical box described in the text for $k_{F}=0.69 \mathrm{fm}^{-1}$. Only the region $0<z<15 \mathrm{fm}, 0<\rho<15 \mathrm{fm}$ is shown. (a) Gap associated with a $v=1$ vortex in the cell without the nucleus. (b) Gap associated with a $v=1$ vortex in the presence of the nucleus. (c) Gap associated with a $v=2$ vortex in the cell without the nucleus. (d) Gap associated with a $v=2$ vortex in the presence of the nucleus. procedure is iterated up to convergence. At each step the gap is obtained self-consistently according to $\Delta=-g[n] \sum_{\alpha} U_{\alpha} V_{\alpha}$, where $g$ is the strength of a pairing contact interaction, which depends on the density $n$. We have adopted the interaction introduced in Ref. [10], corresponding to the values $g[n]=$ $-481\left[1-\left(n / n_{o}\right)^{0.45}\right] \mathrm{MeV} \mathrm{fm}^{3}$, where $n_{o}=0.08 \mathrm{fm}^{-3}$. This interaction reproduces the values of the pairing gap calculated with a bare force (such as the Argonne potential) in uniform neutron matter with the effective mass associated with the SII force.

To limit computational complexity we have not included the spin-orbit interaction term in the calculation of the singleparticle levels. Furthermore, we have constrained the density distribution associated with the protons to be spherical, in keeping with the fact that they are deeply bound. We have considered a closed-shell configuration corresponding to $Z=$ 40 protons, solving the $v=0$ equations in a spherical box of radius $15 \mathrm{fm}$, using the same $\mathrm{SII}$ force as for the neutrons. The effect of the neutrons on the protons has been included after a spherical average of the various neutron distributions has been carried out.

In what follows we shall first discuss the results associated with a neutron density $n_{\mathrm{ext}} \approx 0.012 \mathrm{fm}^{-3}\left(k_{F} \approx 0.69 \mathrm{fm}^{-1}\right)$ far from the nucleus, in the absence of the vortex $(v=0)$. The calculated neutron density is similar to that calculated by Negele and Vautherin [11] for the corresponding (spherically symmetric) Wigner cell. The radius $R$ of the nucleus is about
$7 \mathrm{fm}$, the associated diffusivity being about $0.9 \mathrm{fm}$, and the number of bound neutrons is about 120 . Far from the nucleus the value of the pairing gap $\Delta_{\text {ext }} \approx 2.2 \mathrm{MeV}$ is equal to the value obtained in the case of uniform neutron matter with the adopted pairing interaction, whereas at the nuclear surface $\Delta \approx 1 \mathrm{MeV}$. The strong suppression of the pairing gap inside the nucleus is a consequence of the density dependence of the pairing interaction.

Let us now study the modifications induced by the presence of a vortex $(v=1)$. We first discuss the case of free neutrons, a system that mimics (except at the edges of the cylinder) uniform neutron matter. In Fig. 1(a) we display the associated pairing gap for the case of a $v=1$ vortex. It is seen that $\Delta$ vanishes along the $z$ axis. For small values of $\rho$ $(<3-4 \mathrm{fm})$, the gap increases linearly as a function of $\rho$. Defining the vortex core radius as the value of $\rho$ for which $\Delta\left(\rho_{\text {core }}\right)=$ $\Delta_{\text {ext }} / 2$, one obtains $\rho_{\text {core }} \approx 1.7 \mathrm{fm}$, a value that is expected to scale similarly to the correlation length $\xi \approx \hbar v_{F} / 2 \Delta$ $(\approx 5 \mathrm{fm})$ [12]. For larger values of $\rho$ the gap increases more slowly, gradually approaching the value $\Delta_{\text {ext }}$. We also find a depletion of the density around the vortex axis, similarly to the results of Ref. [12].

We now turn our attention to the case of a $v=1$ vortex pinned on the nucleus. The pairing gap is shown in Fig. 1(b). Comparing with the results displayed in Fig. 1(a) we see that the pairing gap associated with the vortex is strongly influenced by the presence of the nucleus, being strongly suppressed within its volume and also on its surface [e.g., at $\rho \approx 7 \mathrm{fm}$ 


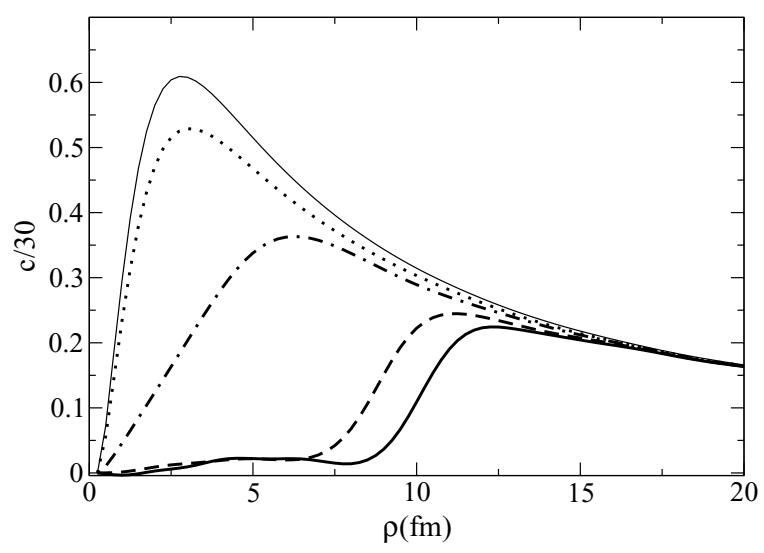

FIG. 2. The velocity associated with the vortex flow (in units of $1 / 30$ times the speed of light $c$ ) shown as a function of $\rho$ for different values of $z: z=0 \mathrm{fm}$ (thick solid line), $z=5 \mathrm{fm}$ (dashed line), $z=10 \mathrm{fm}$ (dash-dotted line), $z=15 \mathrm{fm}$ (dotted line), and $z=$ $17.5 \mathrm{fm}$ (thin solid line).

(and $z=0) \Delta \approx 0.5 \mathrm{MeV}$ ], both compared to the case of an isolated nucleus in the absence of the vortex $(\Delta \approx 1 \mathrm{MeV})$. and compared to the case of a vortex in uniform (neutron) matter [Fig. 1(a); $\Delta \approx 2 \mathrm{MeV}$ ].

The typical linear rise of the gap away from the vortex axis is delayed by about $8 \mathrm{fm}$, which is about the radius of the nucleus, as compared to the uniform case. In other words, the vortex opens and surrounds the nuclear volume, being unable to penetrate into it. The same effect appears in the velocity of the vortex flow, shown in Fig. 2. The velocity vanishes inside the nuclear volume, and the typical rise of the velocity field from zero to the asymptotic Onsager dependence $v=\hbar / 2 m \rho$ is delayed, again by about $8 \mathrm{fm}$ at $z=0$. The vortex induces some changes on the nuclear density, which becomes somewhat elongated along the vortex axis, increases close to the center of the nucleus, and decreases close to the surface, mostly around the equatorial plane.

These results are a consequence of the fact that building a $v=1$ vortex requires the formation of Cooper pairs out of single-particle levels of opposite parity. For not too high values of the Fermi energy, this is strongly hindered inside the nucleus by the spatial quantization associated with finite-size nuclear effects. The latter lead to a distribution of levels around the Fermi energy, essentially displaying either positive or negative parity (in other words, a very small $v=1\left[\pi=(-1)^{v}=-1\right]$ phase space [13]). The consequences of spatial quantization on a $v=1$ vortex can be further clarified by comparing the correspondent solution of the De Gennes equations with that associated with a $v=2$ vortex (see Figs. 1(c) and 1(d); note that within the present context the stability of this solution is immaterial). It is seen that the pairing gap in the case $v=2$ is modified very little by the presence of the nucleus. This is because $v=2$ vortices thrive on a subspace of levels, displaying the same parity.

We now turn our attention to the calculation of the pinning energy $E_{\text {pinn. }}$. This is defined as the difference between the energy cost to create a vortex in between two nuclei (intersti- tial/uniform configuration), $E_{\mathrm{IU}}$, and the energy cost to create a vortex on top of a nucleus (pinned/nuclear configuration), $E_{\mathrm{PN}}: E_{\mathrm{pinn}}=E_{\mathrm{PN}}-E_{\mathrm{IU}}$ [4]. We remark that a negative value of the pinning energy means that the vortex is attracted by the nucleus. To calculate $E_{\mathrm{IU}}$ we shall neglect, following Ref. [4], the influence of the nuclei of the Coulomb lattice on the vortex: as a consequence, $E_{\mathrm{IU}}$ is equal to the energy cost to create an isolated vortex in infinite uniform matter. In practice, we shall compute the difference between the energy $E_{I}$ of a cylinder filled with only neutrons and a vortex along the $z$ axis [cf. Fig. 1(a)] and the total energy $E_{U}$ of the same cylinder with the same number of particles and without a vortex: $E_{\mathrm{IU}}=E_{I}-E_{U}$. Similarly, $E_{\mathrm{PN}}$ will be computed as the difference between the energy $E_{P}$ of a cylinder with a nucleus at its center with a vortex along the $z$-axis [cf. Fig. 1(b)] and the energy $E_{N}$ of the same system without the vortex: $E_{\mathrm{PN}}=E_{P}-E_{N}$. To be meaningful, the cost to create a vortex should refer to two systems with the same number of neutrons moving in the same box. The corresponding solution of the self-consistent Eqs. (1)-(2) leads to a slight change $\left(\delta E_{F} \approx 0.05 \mathrm{MeV}\right)$ of the Fermi energy in going from a system without a vortex to a system with a vortex.

For each of the four configurations, the total energy receives contributions from three sources: the kinetic energy $\left(E_{\mathrm{kin}}\right)$, the mean-field potential (HF) energy $\left(E_{\text {pot }}\right)$, and the pairing energy $\left(E_{\text {pair }}\right)$, which are listed in Table I, from which one obtains the values $E_{\mathrm{IU}}=3834.6-3784.5=50.1 \mathrm{MeV}$ and $E_{\mathrm{PN}}=$ $2966.8-2913.4=53.4 \mathrm{MeV}$. The pinning energy then turns out to be $E_{\text {pinn }}=53.4-50.1=3.3 \mathrm{MeV}$ [14].

Besides the case $k_{F}=0.69 \mathrm{fm}^{-1}\left(n_{\mathrm{ext}}=0.011 \mathrm{fm}^{-3}\right)$ just illustrated, we have calculated the pinning energies at other three densities, corresponding to Fermi momenta $k_{F}=$ $0.34,1.01$, and $1.12 \mathrm{fm}^{-1}$ (or to asymptotic neutron densities $n_{\text {ext }}=1.3 \times 10^{-3}, 3.5 \times 10^{-2}$, and $4.7 \times 10^{-2} \mathrm{fm}^{-3}$ ). The values of $Z$ and the other parameters of the calculation are the same as adopted in the case $k_{F}=0.69 \mathrm{fm}^{-1}$. The four calculations correspond to average baryon densities $n_{b}=1.8 \times 10^{-3}$, $1.3 \times 10^{-2}, 3.8 \times 10^{-2}$, and $5.1 \times 10^{-2} \mathrm{fm}^{-3}$. The qualitative features of this vortex described remain the same. The values of the vortex core radius in uniform matter are $\rho_{\text {core }}=2.7,1.3$, and $4.0 \mathrm{fm}$ for $k_{F}=0.34,1.01$, and $1.12 \mathrm{fm}^{-1}$, respectively; those for the pinned vortex (at $z=0$ ) lie in all cases in the range $9.5-10.5 \mathrm{fm}$. As already stated, we neglect the effect of the vortex on nonpinned, distant nuclei; this requires that $\rho_{\text {core }}$ be much smaller than the internuclear distance. The case $k_{F}=$ $1.2 \mathrm{fm}^{-1}$, for which the Wigner-Seitz radius is about $20 \mathrm{fm}$, lies close to the limit of validity of our approximation. Calculations

TABLE I. The total energy subdivided into $E_{\text {kin }}, E_{\text {pot }}$, and $E_{\text {pair }}$ for each of the four configurations discussed in the text, for $k_{F}=$ $0.69 \mathrm{fm}^{-1}$. All energies are in $\mathrm{MeV}$.

\begin{tabular}{lcccc}
\hline \hline Configuration & $E_{\text {kin }}$ & $E_{\text {pot }}$ & $E_{\text {pair }}$ & Total \\
\hline $\mathrm{U}$ & 6841.9 & -1735.3 & -1322.1 & 3784.5 \\
$\mathrm{I}$ & 6776.0 & -1737.5 & -1203.9 & 3834.6 \\
$\mathrm{~N}$ & 9971.9 & -5784.0 & -1274.5 & 2913.4 \\
$\mathrm{P}$ & 9893.4 & -5806.1 & -1120.5 & 2966.8 \\
\hline \hline
\end{tabular}



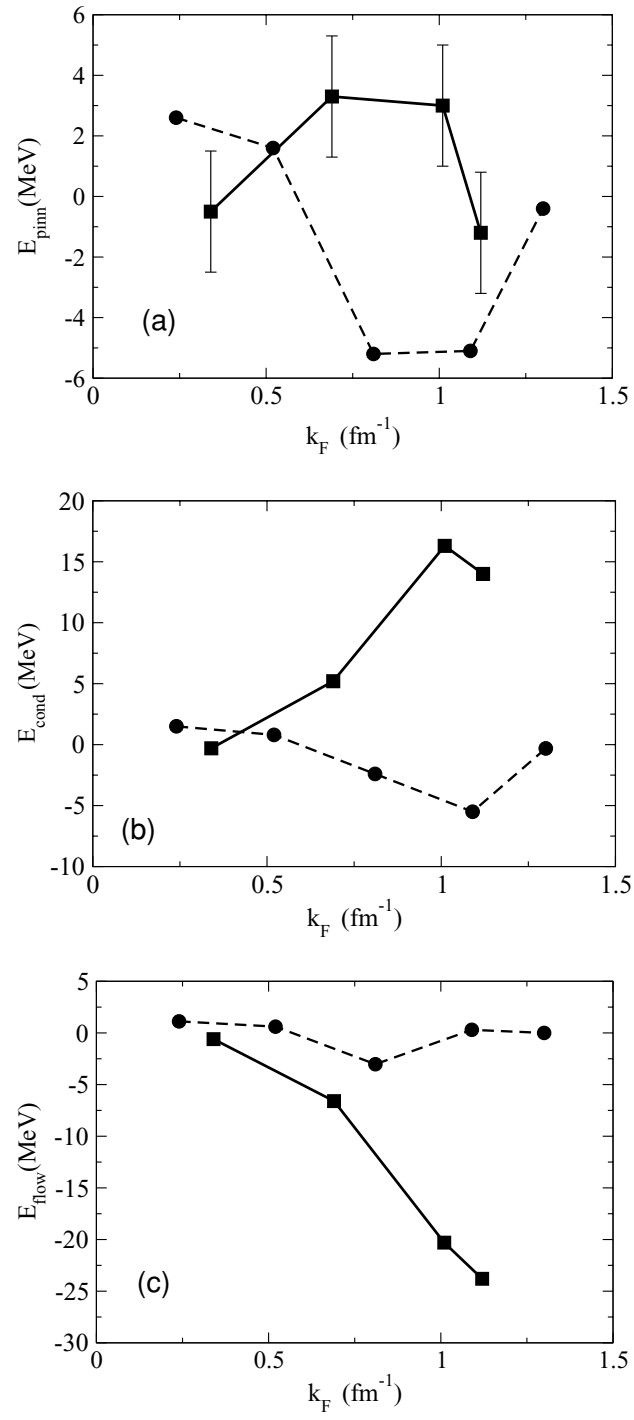

FIG. 3. Pinning energy (a), condensation energy (b), and kinetic energy of the vortex flow (c) as a function of Fermi momentum, according to our quantal model (squares) and to the semiclassical model of Ref. [6] (circles). Also shown is the estimated uncertainty associated with our calculation of the pinning energy.

at higher densities would not be reliable, also because nuclei may acquire a shape different from the spherical one assumed here ("spaghetti" or "lasagna" phase). Our results are shown in Fig. 3(a), where they are compared with the recent findings of Ref. [6], based on a semiclassical approximation. We find that the pinned configuration is favored at the lowest and at the highest Fermi momenta. In contrast, the semiclassical results (in keeping with previous work, cf., e.g., $[4,6,15]$ ) do not show pinning at low density, indicating instead pinning at medium density. To discuss this different dependence on $k_{F}$, it is useful to extract the values of the condensation energy $E_{\text {cond }}$ and of the vortex flow kinetic energy $E_{\text {flow }}$ from our calculation [16]. The main quantity that determines the pinning energy in the semiclassical model is precisely $E_{\text {cond }}$, which is shown in Fig. 3(b). Except at very low density, this quantity is negative, because in the pinned configuration the volume in which one has normal matter is smaller than in the interstitial one: this is related to the fact that $\rho_{\text {core }}(z)$ is little affected by the presence of the nucleus. In our calculation, however, $\rho_{\text {core }}(z)$ becomes much larger, increasing by an amount of the order of the nuclear radius for $z=0$. As a consequence, in the pinned configuration the total volume in which pairing is destroyed is larger than in the interstitial configuration, leading to positive values of $E_{\text {cond }}$. At the same time, the vortex flow is displaced at larger values of $\rho$ (cf. Fig. 2), and this corresponds to smaller velocities and to a reduction of kinetic energy, compared to the interstitial configuration [cf. Fig. 3(c)]. Given the large differences existing in the dependence of $E_{\text {cond }}$ and $E_{\text {flow }}$ on $k_{F}$, it is not surprising that the dependence of $E_{\text {pinn }}$ also turns out to be very different in the two models. Within this context it is useful to remember that, by using a fixed potential, $E_{\text {pinn }}$ can be estimated to a very good accuracy, by simply adding $E_{\text {cond }}$ and $E_{\text {flow }}$. In our self-consistent calculation, it is important to also include the modifications associated with the rearrangement of the neutron density, which contribute to the pinning energy by several $\mathrm{MeV}$. The qualitative features of the results discussed here are expected to be rather independent of the pairing interaction used in the calculations. This is because they are related to very general and well-established properties of nucleons moving in finite nuclei close to the Fermi energy.

We conclude that quantal calculations of vortices in the inner crust of neutron stars lead to a dependence of pinning energy on density, which is qualitatively different from that predicted in all previous work published in the literature. Its astrophysical implications should be assessed by detailed models of vortex dynamics in neutron stars.

Discussions with B. Link are gratefully acknowledged.
[1] The Structure and Evolution of Neutron Stars, edited by D. Pines, R. Tamagaki, and S. Tsuruta (Addison-Wesley, New York, 1992).

[2] B. Link, R. I. Epstein, and G. Baym, Astrophys. J. 403, 285 (1993); M. B. Larson and B. Link, Mon. Not. R. Astron. Soc. 333, 613 (2001); B. Link and C. Cutler, ibid. 336, 211 (2002); M. Hirasawa and N. Shibazaki, Astrophys. J. 563, 267 (2001); P. B. Jones, Phys. Rev. Lett. 79, 792 (1997); 81, 4560 (1998); Mon. Not. R. Astron. Soc. 257, 501 (1992); 296, 217 (1998).

[3] B. Link, Phys. Rev. Lett. 91, 101101 (2003).

[4] R. I. Epstein and G. Baym, Astrophys. J. 328, 680 (1988).
[5] F. V. De Blasio and Ø. Elgaroy, Phys. Rev. Lett. 82, 1815 (1999); $\varnothing$. Elgaroy and F. V. De Blasio, Astron. Astrophys. 370, 939 (2001).

[6] P. M. Pizzochero, L. Viverit, and R. A. Broglia, Phys. Rev. Lett. 79, 3347 (1997); P. Donati and P. M. Pizzochero, Nucl. Phys. A742, 363 (2004).

[7] A. Fabrocini, S. Fantoni, A. Yu. Illarionov, and K. E. Schmidt, Phys. Rev. Lett. 95, 192501 (2005).

[8] P. G. De Gennes, Superconductivity of Metals and Alloys (Addison-Wesley, New York, 1999).

[9] D. Vautherin and D. M. Brink, Phys. Rev. C 5, 626 (1972). 
QUANTUM CALCULATION OF VORTICES IN THE INNER. . .

[10] E. Garrido, P. Sarriguren, E. Moya de Guerra, and P. Schuck, Phys. Rev. C 60, 064312 (1999).

[11] J. W. Negele and D. Vautherin, Nucl. Phys. A207, 298 (1973).

[12] Y. Yu and A. Bulgac, Phys. Rev. Lett. 90, 161101 (2003). We have checked that we reproduce the results found in this reference if we scale the strength of our pairing interaction to have the same asymptotic value of the pairing gap (cf. also Ref. [5]).

[13] The spin orbit interaction will not significantly change the phase space density relevant for vortex formation, because it shifts the partners of the $v=1$ Cooper pairs by about the same amount.

[14] The energy cost to create a vortex depends linearly on the height of the cylindrical box used for the calculation and shows a logarithmic divergence with respect to the radius of the box. We have verified that our results obey this geometrical scaling.
PHYSICAL REVIEW C 75, 012805(R) (2007)

Such dependences, however, disappear when we consider the pinning energy. We have verified that the value of the pinning energy, although obtained from the subtraction of large numbers, is remarkably stable with respect to changes in the box size for sufficiently large boxes. We have evaluated the error associated with the finite mesh size used in integrating these equations $(\Delta \rho=\Delta z=0.25 \mathrm{fm})$, and we estimate that the absolute value of the error associated with this quantity is less than $2 \mathrm{MeV}$.

[15] M. A. Alpar et al., Astrophys. J. 346, 823 (1989).

[16] The energy $E_{\text {flow }}$ is obtained integrating the associated kinetic energy density $(1 / 2) \Phi^{2} / n$, where $\Phi$ is the vortex flow, $\Phi=$ $\hbar / m \sum_{\alpha} V_{\alpha}^{2}(\rho, z)\left(l_{\alpha}-v\right)$. The condensation energy $E_{\text {cond }}$ is obtained by integrating the density $-(3 / 8) n(\rho, z) \Delta^{2}(\rho, z) /\left[E_{F}-\right.$ $V(\rho, z)]$. 